\title{
Investigating the level of agreement of two positioning protocols when using Dual energy X-ray absorptiometry in the assessment of body composition
}

\author{
Flinn Shiel ${ }^{1}$, Carl Persson $^{1}$, Vini Simas ${ }^{2}$, James Furness ${ }^{\text {Corresp., }}{ }^{1,2}$, Mike Climstein ${ }^{2,3}$, Ben Schram ${ }^{1,2}$ \\ 1 Department of Physiotherapy, Faculty of Health Sciences, Bond University, Robina, Queensland, Australia \\ 2 Water Based Research Unit, Faculty of Health Sciences, Bond University, Robina, Queensland, Australia \\ 3 Exercise Health \& Performance Faculty Research Group, The University of Sydney, Lidcombe, New South Wales, Australia \\ Corresponding Author: James Furness \\ Email address: jfurness@bond.edu.au
}

Background. Dual energy X-ray absorptiometry (DXA) is a commonly used instrument for analysing segmental body composition (BC). The information from the scan guides the clinician in the treatment of conditions such as obesity and can be used to monitor recovery of lean mass following injury. Two commonly used DXA positioning protocols have been identified - the Nana positioning protocol and the National Health and Nutrition Examination Survey (NHANES). Both protocols have been shown to be reliable. However, only one study has assessed the level of agreement between the protocols and ascertained the participants' preference of protocol based upon comfort. Given the paucity of research in the field and the growing use of DXA in both healthy and pathological populations further research determining the most appropriate positioning protocol is warranted. Therefore, the aims of this study were to assess the level of agreement between results from the NHANES protocol and Nana protocol, and the participants' preference of protocol based on comfort.

Methods. Thirty healthy participants (15 males, 15 females, aged 23 to 59 years) volunteered to participate in this study. These participants underwent two whole body DXA scans in a single morning (Nana positioning protocol and NHANES positioning protocol), in a randomised order. Each participant attended for scanning wearing minimal clothing and having fasted overnight, refrained from exercise in the past $24 \mathrm{hrs}$ and voided their bladders. Level of agreement, comparing NAHNES to Nana protocol was assessed using an intra-class correlation coefficient (ICC), concordance correlation coefficient (CCC) and percentage change in mean. Limit of agreement comparing the two protocols were assessed using plots, mean difference and confidence limits. Participants were asked to indicate the protocol they found most comfortable.

Results. When assessing level of agreement between protocols both the ICC and CCC scores were very high and ranged from 0.987 to 0.997 for whole body composition, indicating excellent agreement between the Nana and NHANES protocols. Regional analysis (arms, legs, trunk) ICC scores, ranged between 0.966 to 0.996, CCC ranged between 0.964 and 0.997 , change in mean percentage ranged between $-0.58 \%$ and $0.37 \%$ which indicated a very high level of agreement. Limit of agreement analysis using mean difference ranged between -0.223 and $0.686 \mathrm{~kg}$ and $95 \% \mathrm{CL}$ produced results ranging between $-1.262 \mathrm{~kg}$ and $1.630 \mathrm{~kg}$. The majority (80\%) of participants found the NHANES positioning protocol more comfortable.

Discussion. This study reveals a strong level of agreement as illustrated by high ICC's and CCC'S between the positioning protocols, however systematic bias within limit of agreement plot and a large 
difference in $95 \%$ confidence limits indicates that the protocols should not be interchanged when assessing an individual. The NHANES protocol affords greater participant comfort. 
$4 \quad$ Investigating the level of agreement of two positioning protocols

5 when using Dual energy $X$-ray absorptiometry in the assessment of body composition.

8 AUTHORS

9 Flinn Shiel ${ }^{1}$, Carl Persson ${ }^{1}$, Vini Simas ${ }^{2}$, James Furness ${ }^{1,2}$, Ben Schram ${ }^{1,2}$, Mike Climstein ${ }^{2,3}$

$11{ }^{1}$ Physiotherapy Department, Faculty of Health Sciences and Medicine, Bond University, Robina, 12 QLD, Australia

132 Water Based Research Unit, Faculty of Health Sciences and Medicine, Bond University, 14 Robina, QLD, Australia

$15{ }^{3}$ Exercise Health \& Performance Faculty Research Group, Faculty of Health Sciences, The 16 University of Sydney, Lidcombe, NSW, Australia

17

18

19 Corresponding Author:

20 Dr James Furness

21 Email address: jfurness@bond.edu.au 


\section{ABSTRACT}

26 Background. Dual energy X-ray absorptiometry (DXA) is a commonly used instrument for 27 analysing segmental body composition (BC). The information from the scan guides the clinician 28 in the treatment of conditions such as obesity and can be used to monitor recovery of lean mass 29 following injury. Two commonly used DXA positioning protocols have been identified - the 30 Nana positioning protocol and the National Health and Nutrition Examination Survey 31 (NHANES). Both protocols have been shown to be reliable. However, only one study has 32 assessed the level of agreement between the protocols and ascertained the participants' 33 preference of protocol based upon comfort. Given the paucity of research in the field and the 34 growing use of DXA in both healthy and pathological populations further research determining 35 the most appropriate positioning protocol is warranted. Therefore, the aims of this study were to 36 assess the level of agreement between results from the NHANES protocol and Nana protocol, 37 and the participants' preference of protocol based on comfort.

38 Methods. Thirty healthy participants (15 males, 15 females, aged 23 to 59 years) volunteered to 39 participate in this study. These participants underwent two whole body DXA scans in a single 40 morning (Nana positioning protocol and NHANES positioning protocol), in a randomised order.

41 Each participant attended for scanning wearing minimal clothing and having fasted overnight, 42 refrained from exercise in the past $24 \mathrm{hrs}$ and voided their bladders. Level of agreement, 43 comparing NAHNES to Nana protocol was assessed using an intra-class correlation coefficient 44 (ICC), concordance correlation coefficient (CCC) and percentage change in mean. Limit of 
45

46

47

48

49

50

51

52

53

54

55

60

61

62

63

64

65 67 66 development and implementation of the DXA scanner (Lewiecki, 2005). Dual energy X- ray

agreement comparing the two protocols were assessed using plots, mean difference and confidence limits. Participants were asked to indicate the protocol they found most comfortable.

Results. When assessing level of agreement between protocols both the ICC and CCC scores were very high and ranged from 0.987 to 0.997 for whole body composition, indicating excellent agreement between the Nana and NHANES protocols. Regional analysis (arms, legs, trunk) ICC scores, ranged between 0.966 to 0.996 , CCC ranged between 0.964 and 0.997 , change in mean percentage ranged between $-0.58 \%$ and $0.37 \%$ which indicated a very high level of agreement. Limit of agreement analysis using mean difference ranged between -0.223 and $0.686 \mathrm{~kg}$ and $95 \%$ CL produced results ranging between $-1.262 \mathrm{~kg}$ and $1.630 \mathrm{~kg}$. The majority $(80 \%)$ of participants found the NHANES positioning protocol more comfortable.

Discussion. This study reveals a strong level of agreement as illustrated by high ICC's and CCC's between the positioning protocols, however systematic bias within limit of agreement plot and a large difference in $95 \%$ confidence limits indicates that the protocols should not be interchanged when assessing an individual. The NHANES protocol affords greater participant comfort.

\section{INTRODUCTION}

3 Tissue composition assessment and analysis is commonly undertaken by using dual-energy X4 ray absorptiometry (DXA) (Nana et al., 2012). The need for a device to accurately and reliably 5 measure bone mineral density as an indicator of an individual's bone health, led to the 7 absorptiometry emits energy sources that are absorbed at different degrees of attenuation relative 
68 to the type of tissue they encounter; thus enabling clear imagining of different tissues (fat mass,

69 lean mass and bone) based upon the distinctive elements of these tissues (Rothney et al., 2009).

70 Due to these distinct properties of measurement, the DXA scan calculates an individual's total

71 body composition (BC), together with an individual's regional BC; thus, the DXA is a popular

72 instrument in research and clinical settings. Furthermore, DXA produces $0.004 \mathrm{mSv}$ of radiation

73 in each $\mathrm{BC}$ scan, equating to less than $1 \%$ of the maximum radiation dosage of $5 \mathrm{mSv}$ in a year,

74 as described by Australian Radiation Protection and Nuclear Safety Agency (ARPANSA, 2005).

75 Therefore, the minimal level of radiation from DXA scans enables researchers and clinicians to

76 widely use this instrument to assess $\mathrm{BC}$ on a regular basis. Research drawn from $\mathrm{BC}$ scans has

77 assisted clinicians and researchers to further their understanding of a number of conditions,

78 including obesity and undernourished individuals (Lee \& Gallagher, 2008). When applying BC

79 scanning to athletes, it has been identified that those with higher muscle mass in pre-season, have

80 a decreased likelihood of suffering bone related injuries during the season (Georgeson et al.,

81 2011). Nevertheless, it is important to note that the DXA's reliability must be ascertained prior to

82 statistical data being extracted, analysed and applied within a clinical and or sporting 83 population.

84

85 In previous studies a variety of statistical analysis methods have been undertaken including intra86 class correlation coefficients (ICC), percentage change and Pearson correlations to assess the 87 reliability of the DXA, all of which have found DXA to be reliable (Bilsborough et al., 2014; 88 Climstein et al., 2015; Colyer et al., 2016; Covey et al., 2010; Covey et al., 2008; Kerr et al., 89 2016; Lohman et al., 2009; Moon et al., 2013; Nana et al., 2012; Nana et al., 2013; Smith-Ryan 90 et al., 2016). However higher reliability is found in studies that account for biological and 
91 technical errors, especially the use of a reproducible positioning protocol. The National Centre

92 for Health Statistics, National Health and Nutrition Examination Survey (NHANES) body 93 composition positioning protocol (NHANES, 2013) and the Nana positioning protocol, founded

94 by Alisa Nana, are the two most popularly used protocols (Nana et al., 2012). It is important to 95 note the Australian and New Zealand Bone Mineral Society (ANZBMS) employs the same body 96 position as the NHANES positioning protocol.

98 Shiel et al. (unpublished data) have systematically assessed studies using the Nana and NHANES 99 positioning protocols and concluded that there is a high level of evidence and excellent reliability 100 for the Nana positioning protocol, and a moderate level of evidence but excellent reliability for 101 the NHANES, and therefore the Nana protocol should be considered the gold standard for BC 102 DXA scanning. Kerr et al., (2016) is the only study to date which has compared the Nana and 103 NHANES positioning protocols; concluding that the Nana protocol's reliability is superior in 104 assessment of regional $\mathrm{BC}$, fat mass (FM) and bone mineral content (BMC). This study also 105 recommended that positioning protocols should not be interchanged, and proposes that the Nana 106 positioning protocol is the more comfortable for the participant (Kerr et al., 2016). However, it 107 should be noted that the Kerr study has used modified versions of the original protocols, which 108 may have altered the participants perceived comfort level during the scan.

110 As such the primary aim of our study is to conduct an independent comparison of the Nana and 111 NHANES positioning protocols in terms of results and level of agreement. The finding of this 112 research will either strengthen the findings suggesting the Nana protocol produces superior 113 results or increase the level of evidence for the NHANES protocol. Additionally, this study 
114 aimed to assess which of the two main positioning protocols identified in the published literature

115 is more comfortable.

116

117 METHODS

118

119 Study overview

120 During a single session, each participant underwent a total body scan twice, being repositioned

121 between each scan. The two scans consisted of one using the Nana positioning protocol, with

122 feet and hands positioned in radio-opaque pads; the other scan utilized the NHANES positioning

123 protocol scan, where the hands are positioned faced down on the scanning bed. The order of the

124 positioning protocol scans was randomised. Each participant was asked to choose which

125 positioning protocol, Nana or NHANES, was the most comfortable, and why they selected that

126 positioning protocol.

\section{Participants}

129 Fifteen males and fifteen females $(n=30)$ were recruited from the local university and the greater

130 public to partake in this comparative study. Thirty participants were selected based upon the

131 previously published recommendations for reliability studies (Lexell \& Downham, 2005).

132 Participants underwent an anthropometrical analysis of height (to the closest $0.1 \mathrm{~cm}$ ) using a

133 medical stadiometer (Harpenden, Holtain Limited, Crymych, UK) and mass (to the closest

$1340.1 \mathrm{~kg}$ ) on medical scales (WM202, Wedderburn, Bilinga, Australia) prior to undergoing a BC

135 scan on the DXA. Participant characteristics can be found in Table 1. Prior to partaking in the

136 study, all participants were informed of the testing procedures and signed a consent form. The 
137 study was granted ethics approval by Bond University Human Research Ethics Committee

138 (R015221).

Table 1. Participant Characteristics

\begin{tabular}{llll}
\hline & Males $(\mathrm{n}=15)$ & Females $(\mathrm{n}=15)$ & Group $(\mathrm{n}=30)$ \\
\hline Age $(\mathrm{yr})$ & $27.8 \pm 7.2$ & $31.3 \pm 11.9$ & $29.6 \pm 10.1$ \\
Height $(\mathrm{cm})$ & $178.7 \pm 7.3$ & $164.7 \pm 8.9$ & $171.7 \pm 10.7$ \\
Mass $(\mathrm{kg})$ & $78.9 \pm 8.8$ & $62.4 \pm 9.7$ & $70.6 \pm 12.4$ \\
\hline
\end{tabular}

139

140 Standardised Baseline Conditions

141 On the morning of the scan, the participant confirmed they had fasted overnight; rested and

142 refrained from strenuous exercise for the previous 24hrs; wore minimal clothing (males:

143 underwear, females: underwear, sports bra or two piece bathers); bladder voided; as well as

144 jewellery and metal removed, prior to scanning.

145

146 DXA instrument

147 BC was measured using a narrow angle fan beam Lunar Prodigy DXA machine (GE Healthcare, 148 Madison, WI) with automatic analysis performed using GE enCore 2016 software (GE

149 Healthcare). DXA provides three-component approximation of bone tissue and soft tissue (lean 150 tissue i.e. muscle) and fat tissue (ANZBMS, 2014). The DXA was calibrated daily prior to any 151 scans using a phantom as per manufacturer's guidelines. The machine used for the study has 152 previously been found to produce very high reliability for BMD (0.998), lean mass (0.989) 153 and fat mass (0.995) (Climstein et al., 2015).

154

155 Standardised DXA operational protocol

156 All scans were performed by the same licensed researcher with all scans analysed automatically 157 by the GE enCORE 2016 software. Two BC protocols were utilised, the NHANES positioning 
158 protocol and the Nana positioning protocol (Figure 1). The NHANES protocol required the 159 participant to be positioned in a supine position in the middle of the densitometry table with head 160 straight, space between the arms and torso, palms flat on the table, and feet together secured by a 161 strap (NHANES, 2013). When utilising the Nana positioning protocol, participants were 162 centrally aligned in the scanning area with their feet placed in a custom-made foam block to 163 maintain a consistent distance between the subject's feet $(15 \mathrm{~cm})$ in each scan. The custom-made 164 foot blocks were made from styrofoam and were transparent under the DXA scan. Additionally, 165 the subject's hands were placed in custom-made foam and plastic paddles to ensure a mid-prone 166 position with a standardised gap $(3 \mathrm{~cm})$ between the palms and trunk. These hand paddles 167 created minimal changes to the scan analysis (Nana et al., 2012). Additionally, a strap around the 168 ankles was utilised as per the NHANES protocol, to ensure that the only difference between 169 protocols was the positioning block/paddles. 


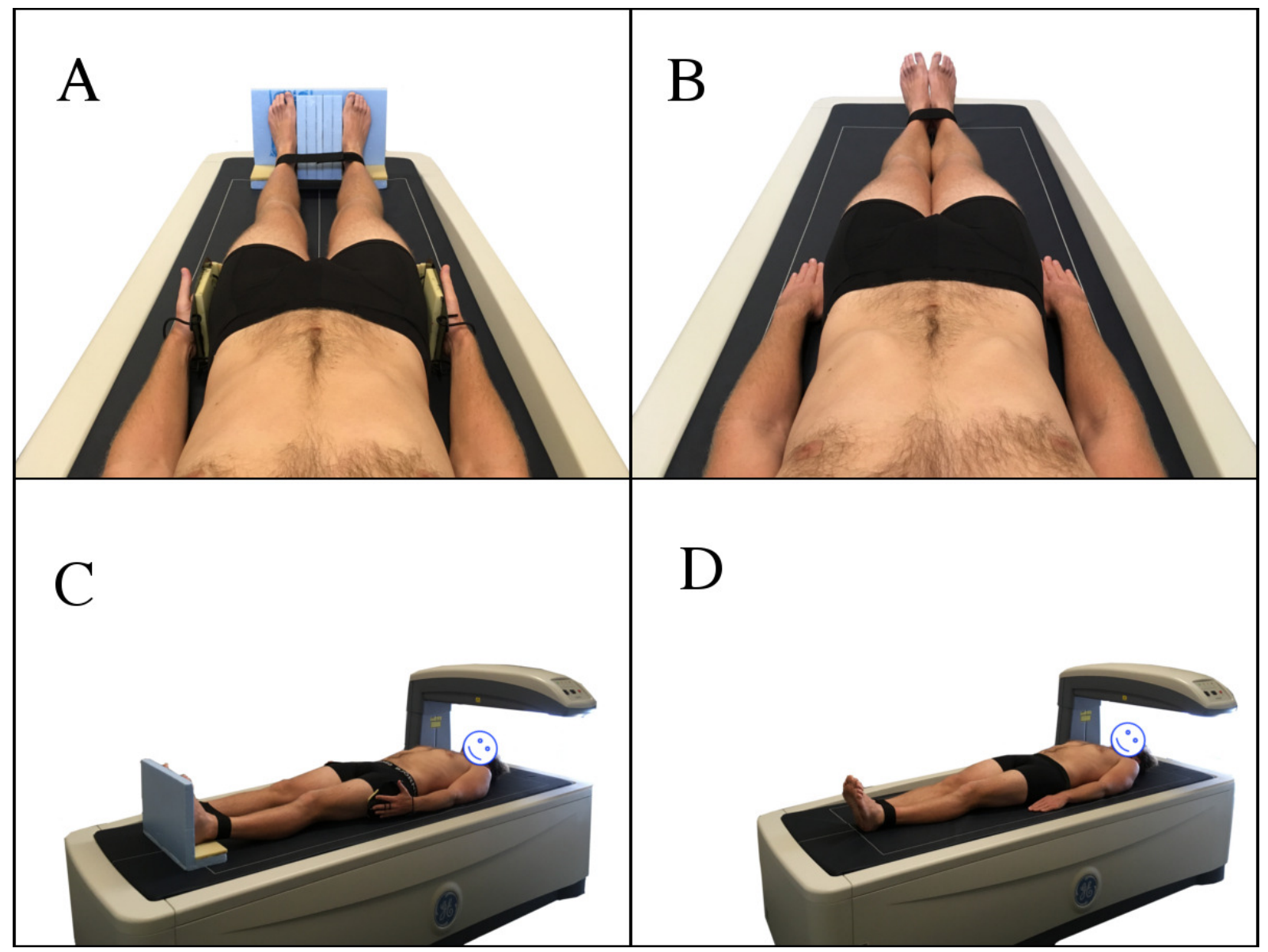

171 Figure 1. Nana positioning protocol $(A, C)$ and NHANES positioning protocol $(B, D)$

\section{Statistical Analysis}

174 IBM SPSS (version 24.0) and a custom reliability spreadsheet from Sportscience web site

175 (www.sportsci.org) were used to analyse the data. Anthropometrical data were presented as

176 means and standardised deviations. IBM SPSS 24 was utilised to assess Intra-class Correlation

177 Coefficient $(3,1)$ with Confidence Intervals (CI), Concordance Correlation Coefficient (CCC)

178 with 95\% Confidence Limits (CL) and create Limit of Agreement analysis plots and assess mean

179 difference and associated confidence limits. This specific ICC was selected, based on the

180 published work of Trevethan (Trevethan, 2016). Percentage change in mean and typical error 
181 expressed as coefficient of variation as a percentage (CV\%) were calculated using the

182 customised Sportscience spreadsheet.

183

184 RESULTS

185

186 All results comparing the Nana positioning protocol with the NHANES positioning protocol

187 (Figure 2) are presented in Table 2. When assessing the BC using two different positioning 188 protocols; the results of the whole body (tissue, FM, LM and BMC) scans and all regional (arms,

189 legs and trunk) scans were excellent based on ICC's and percentage change in mean statistics.

190 The results are also illustrated in the Limit of Agreement analysis plots for whole body (Figure

1913 ) and Table 3 for all regions.

192

193 Percentage change in mean when comparing the two protocols has produced results that range

194 between $-0.68 \%$ and $0.37 \%$. Trunk was the regional area with the smallest variance of the four 195 sites (whole body, arms, legs and trunk) as described in Table 2, with results ranging from $0.02 \%$ 196 to $0.37 \%$. Whole body scans produced the largest variance, with results ranging from $-0.68 \%$ to $197 \quad 0.21 \%$.

198

199 The typical error expressed as CV\% of the agreement between the positioning protocols and 200 produced results ranging between $0.01 \%$ and $0.42 \%$. The parameter of BMC was assessed to 201 produce the smallest typical error across the four different sites (whole body, arms, legs and 202 trunk). The tissue parameter was found to be the highest in three of four assessment sites (arms, 203 legs and trunk). 
205 A very high level of agreement between the two positioning protocols is evident through an ICC 206 ranging between $0.966-0.999$. Whole body fat mass-produced the highest ICC of 0.997 , with a $20795 \%$ CI of $0.992-0.999$. The fat mass of the arms produced the lowest ICC of 0.966 , with a $20895 \%$ CI of $0.923-0.984$.

209

210 Additional to the ICC, the CCC illustrates very good results with the results ranging between 2110.964 and 0.997 . The whole body lean mass produced the highest result of 0.997 with $95 \%$ CL of $2120.995-0.998$. Similar to the ICC result the fat mass of the arms produced the lowest correlation 213 of 0.964 with $95 \%$ CL $0.936-0.980$.

214

215 Limit of Agreement analysis plots (Figure 3) for the whole body reveal a bias between the two 216 measures when assessing tissue as the zero value lies outside of the interval. This indicates that 217 the Nana protocol consistently produced larger values than the NHANES protocol. Limit of 218 agreement analysis using mean difference between the protocols ranged between -0.223 and $2190.686 \mathrm{~kg}$ across the parameters with arm measures the smallest difference. The 95\% CL produced 220 results ranging from $-1.262 \mathrm{~kg}$ for the lower limit up to $1.630 \mathrm{~kg}$ for the upper limit. All mean 221 differences fell with the define CL except for the leg fat assessment.

223 When questioned about which protocol was the more comfortable, 24 out of 30 participants $224(80.0 \%)$ chose the NHANES positioning protocol as the more comfortable of the two protocols 225 assessed. 

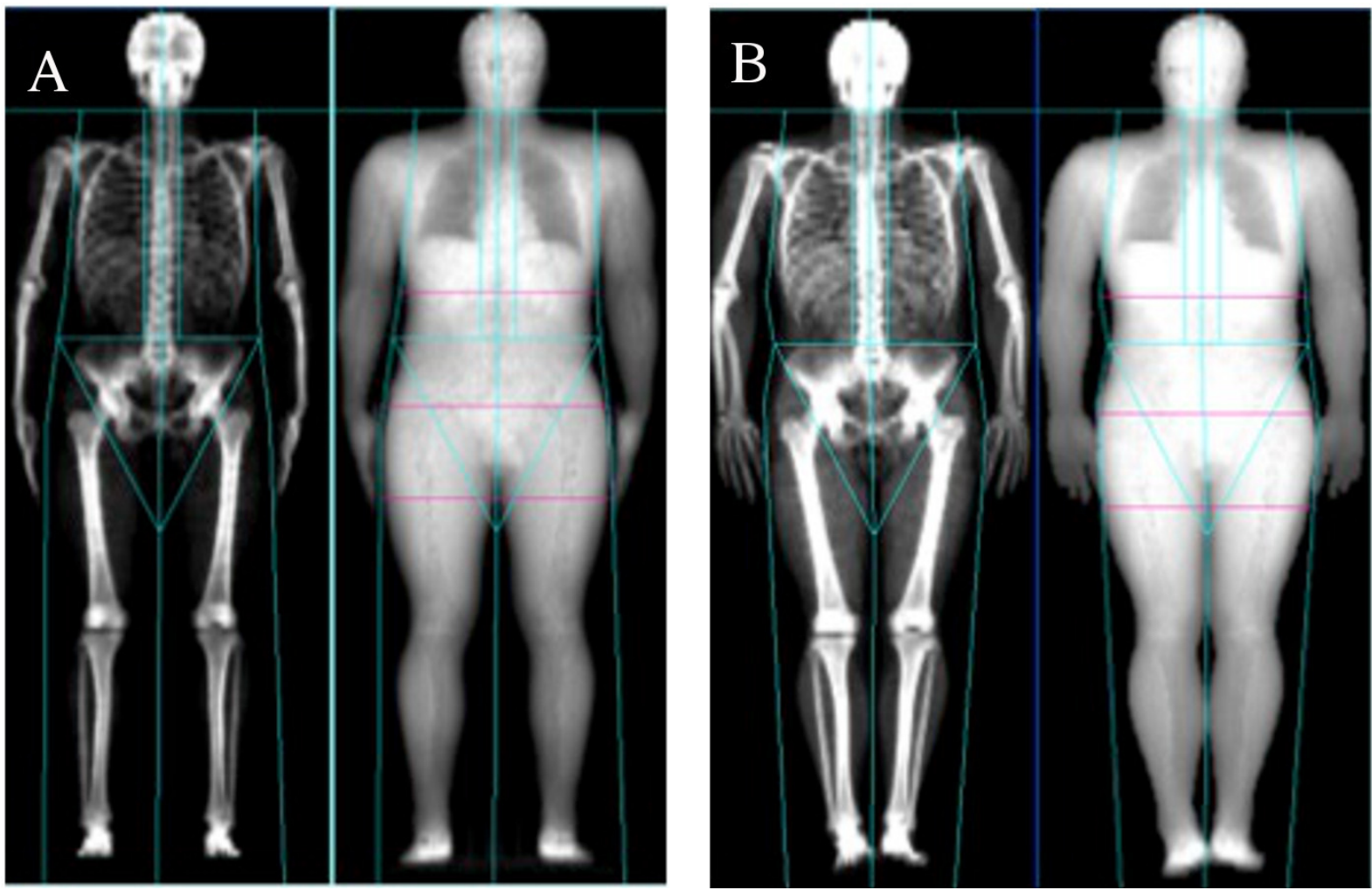

Figure 2. Nana positioning protocol (A) and NHANES positioning protocol (B)

231 Table 2. Level of agreement between Nana vs NHANES positioning protocols

\begin{tabular}{|c|c|c|c|c|c|c|c|}
\hline & & $\begin{array}{c}\% \Delta \text { in } \\
\text { mean }\end{array}$ & $\begin{array}{l}\text { Typical } \\
\text { error as } \\
\text { CV\% }\end{array}$ & ICC & CI $(95 \%)$ & $\mathrm{CCC}$ & CL (95\%) \\
\hline \multirow{4}{*}{$\begin{array}{l}\text { Whole } \\
\text { Body }\end{array}$} & Tissue & -0.47 & 0.10 & 0.987 & $0.970-0.994$ & 0.987 & $0.976-0.993$ \\
\hline & Fat & 0.21 & 0.30 & 0.997 & $0.992-0.999$ & 0.997 & $0.994-0.998$ \\
\hline & Lean & -0.68 & 0.32 & 0.997 & $0.905-0.999$ & 0.997 & $0.995-0.998$ \\
\hline & $\mathrm{BMC}$ & 0.06 & 0.03 & 0.990 & $0.586-0.998$ & 0.989 & $0.983-0.994$ \\
\hline \multirow{4}{*}{ Arms } & Tissue & -0.32 & 0.19 & 0.982 & $0.745-0.995$ & 0.982 & $0.968-0.989$ \\
\hline & Fat & 0.08 & 0.13 & 0.966 & $0.923-0.984$ & 0.964 & $0.936-0.980$ \\
\hline & Lean & -0.39 & 0.15 & 0.980 & $0.329-0.996$ & 0.980 & $0.966-0.980$ \\
\hline & $\mathrm{BMC}$ & 0.01 & 0.01 & 0.979 & $0.876-0.993$ & 0.994 & $0.989-0.997$ \\
\hline \multirow{4}{*}{ Legs } & Tissue & -0.58 & 0.38 & 0.984 & $0.822-0.995$ & 0.983 & $0.971-0.990$ \\
\hline & Fat & -0.10 & 0.19 & 0.992 & $0.983-0.996$ & 0.992 & $0.986-0.996$ \\
\hline & Lean & -0.49 & 0.30 & 0.987 & $0.837-0.996$ & 0.987 & $0.977-0.992$ \\
\hline & $\mathrm{BMC}$ & 0.02 & 0.01 & 0.996 & $0.795-0.999$ & 0.997 & $0.998-0.999$ \\
\hline \multirow{4}{*}{ Trunk } & Tissue & 0.37 & 0.42 & 0.993 & $0.977-0.997$ & 0.993 & $0.987-0.996$ \\
\hline & Fat & 0.22 & 0.29 & 0.991 & $0.975-0.996$ & 0.991 & $0.983-0.995$ \\
\hline & Lean & 0.18 & 0.39 & 0.993 & $0.986-0.997$ & 0.993 & $0.988-0.996$ \\
\hline & $\mathrm{BMC}$ & 0.02 & 0.02 & 0.973 & $0.841-0.991$ & 0.972 & $0.951-0.984$ \\
\hline
\end{tabular}

$232 \% \Delta$ in Mean - percentage change in mean, $\mathrm{CV}$ - confidence variance, ICC - intra-class 
233 correlation coefficient, $\mathrm{CI}$ - confidence interval, CCC - concordance correlation coefficient, CL 234 - confidence limit.

235

236 Table 3. Limit of agreement between Nana vs NHANES positioning protocols

\begin{tabular}{|lcccc|}
\hline & & Mean Difference & Lower CL & Upper CL \\
\hline \multirow{4}{*}{ Whole } & Tissue & 0.473 & -0.191 & 0.756 \\
Body & Fat & -0.212 & -0.621 & 1.044 \\
& Lean & 0.686 & 0.202 & 1.574 \\
& BMC & -0.063 & -0.133 & 0.008 \\
\hline \multirow{4}{*}{ Arms } & Tissue & 0.321 & 0.193 & 0.836 \\
& Fat & -0.074 & -0.432 & 0.283 \\
& Lean & 0.396 & 0.014 & 0.807 \\
& BMC & 0.000 & -0.020 & 0.021 \\
\hline \multirow{6}{*}{ Legs } & Tissue & 0.586 & 0.458 & 1.630 \\
& Fat & 0.099 & 0.420 & 0.618 \\
& Lean & 0.488 & 0.350 & 1.327 \\
& BMC & -0.005 & -0.030 & 0.020 \\
\hline \multirow{6}{*}{ Trunk } & Tissue & 0.366 & 0.806 & 1.538 \\
& Fat & -0.223 & -1.017 & 0.572 \\
& Lean & -0.176 & -1.262 & 0.911 \\
\hline
\end{tabular}

237 CL - Confidence Limit (95\%) 
A

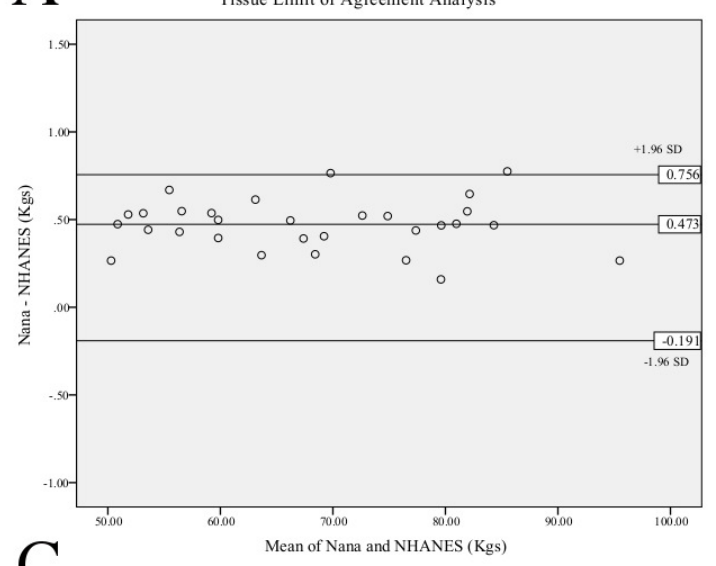

C

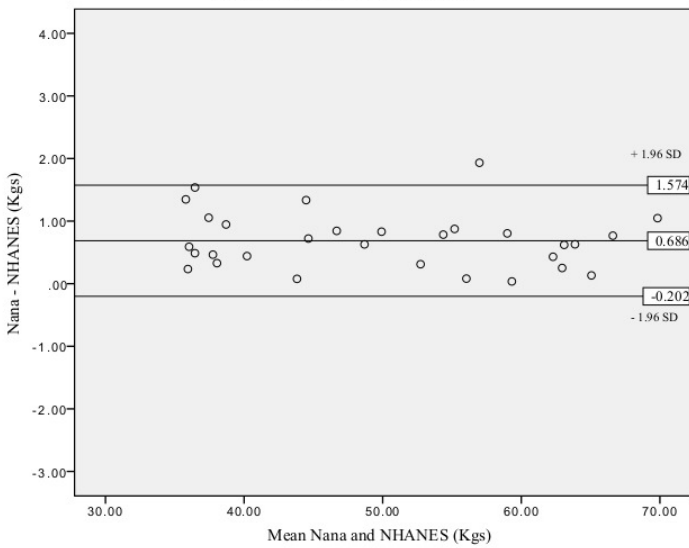

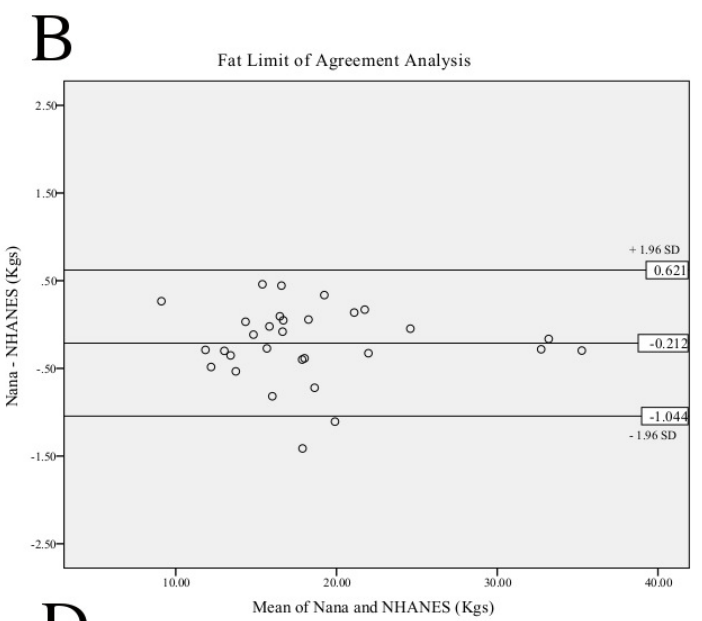

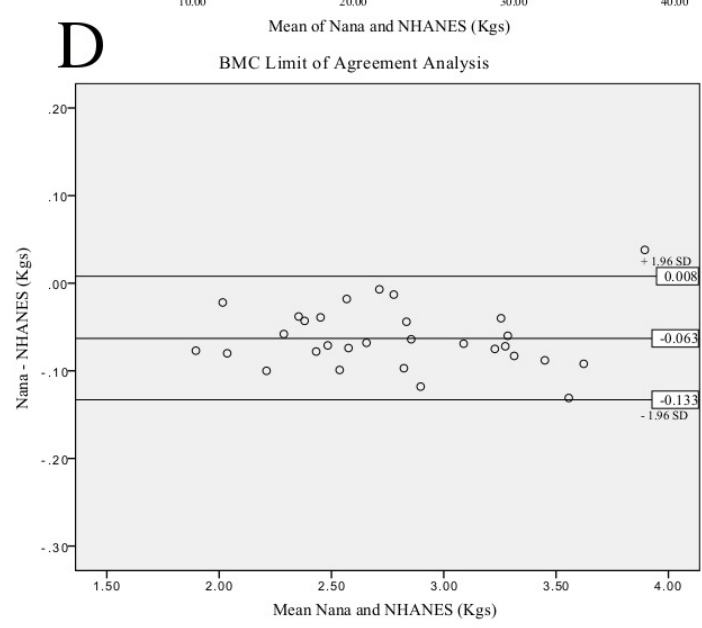

239

Figure 3. Limit of Agreement analysis for Nana versus NHANES whole body positioning protocols. Tissue analysis (A), fat analysis (B), lean analysis (C), BMC analysis (D)

\section{DISCUSSION}

The primary aim of this study was to focus upon technical error associated with positioning and establish the level of agreement between the two identified positioning protocols. This study also sought to identify which DXA scan positioning protocol was the more comfortable for participants. In this study, we conducted all scans of BC using a Lunar DXA machine, located at Bond Institute of Health \& Sport. To minimise the chance of technical error, one licensed researcher (qualified through ANZBMS) conducted all thirty scans as recommended for 
251 reliability studies (Lexell \& Downham, 2005). To further decrease the chance of error affecting

252 the results, biological factors such as time of day of scanning, hydration, exercise and food

253 metabolism have been identified and accounted for.

254

255 This study found that the level of agreement between the Nana and the NHANES positioning 256 protocols was very high when using a variety of statistics including percentage change in mean, 257 accompanied with typical error, or ICC, accompanied with CI. The percentage change in mean 258 findings of this study for the whole body (tissue $-0.47 \%$, FM $0.21 \%$, LM $-0.68 \%$, BMC $0.06 \%$ ) 259 is similar to the results of the previous study comparing the two protocols (tissue $-0.4 \%$, FM $2602.8 \%$, LM $0.3 \%$, BMC $-0.7 \%$ ) (Kerr et al., 2016). The results of this study suggest that the level 261 of agreement between the two protocols when doing regional analysis is also very good however 262 these results are opposed to previously published research that conclude there is a large 263 difference between protocol results (Kerr et al., 2016).

264

265 The assessed percentage change in mean in this study is smaller across the all parameters 266 assessed except for whole body tissue mass in comparison to the only other study that has 267 compared the two positioning protocols (Kerr et al., 2016). This may be due to the stringent 268 methodology used in our study. As both studies have accounted for biological factors creating 269 errors the source of difference can only be technical error. As such in this study, the NHANES 270 protocol was followed as prescribed in NHANES Body Composition Procedures Manual 2013

271 (NHANES, 2013). The participant's feet were secured together with a strap and the hands were

272 placed in a pronated position (palms down on the table), reducing the likelihood of movement 273 artifacts. In comparison, the previous research conducted by Kerr and colleagues, the legs were 
274 secured with a strap but positioned a significant distance apart, possibly allowing for small

275 amounts of internal rotation and adduction as these movements were not limited. Furthermore,

276 the hands were held in a neutral position, possibly allowing for small rotational movements. The

277 combination of these two adjustments to the prescribed NHANES positioning protocol could 278 possibly have created movement artifacts and altered results.

279

280 This is the first study to use an ICC to assess the level of agreement between the two positioning 281 protocols. Very high ICC results are deemed to be between 0.90 and 1.00 (Munro \& 282 Visintainer, 2005), and our results (0.996 - 0.999) fall within this described range. Additionally, 283 the concordance correlation results $(0.964-0.997)$ coupled with the ICC results indicated that 284 the level of agreement between the two positioning protocols is very high, however this needs to 285 be coupled with the mean difference and confidence limits analysis before deciding if the 286 protocols are interchangeable.

287

288 The limits of agreement between the two positioning protocols when plotted into limit of 289 agreement analysis plots (Figure 3) reveals a systematic bias in the parameter of whole body 290 tissue. The systematic bias illustrates that the Nana protocol consistently produces higher results 291 than the NHANES protocol, possibly due to the use of the foam blocks used to secure the feet. 292 Additionally, Table 3 reveals that the mean difference lies outside of the defined 95\% confidence 293 limits for the leg fat parameter, this is due to this parameter having a large difference between the 294 standard deviation and the mean when comparing the protocols. Applying the limit of agreement 295 findings clinically illustrates a large variance, for example if the participant's lean mass was $29650 \mathrm{~kg}$ and mean difference $1.75 \mathrm{~kg}$ then this equates to $4 \%$ change. These factors indicate that the 
297 two positioning protocols should not be used interchangeably even though the ICC results are 298 very high.

299

300 When assessing which positioning protocol (Nana or NHANES) was deemed the most 301 comfortable, this study found that 24 out of 30 participants $(80.0 \%)$ chose the NHANES 302 positioning protocol to be the most comfortable, this result is in direct opposition to previous 303 findings (Kerr et al., 2016). Upon closer inspection of the methods employed, it appears Kerr and 304 colleagues altered the original NHANES and Nana positioning protocols, which would have 305 affected the perceived comfort levels of participants. The modified version of the NHANES 306 positioning protocol they employed, would have required muscular activation and control; 307 therefore, decreasing the participant's perceived comfort. When using the Nana positioning 308 protocol, a strap was added to the original Nana protocol, which secured the participants arms for 309 approximately seven minutes during scanning; hence decreasing the muscular activation and 310 increasing the participant's perceived comfort. In our study, the majority of participants who 311 chose the NHANES as the most comfortable did so, because they felt their hands and arms were 312 in a more relaxed position.

314 The Nana positioning protocol, where the feet are placed in radio-opaque blocks to maintain 315 plantargrade ankle position; allows for taller individuals to be scanned with a decreased risk of 316 plantar flexion and the participant's feet moving outside the scanning field (Nana et al., 2012). 317 Most individuals in our study over the height of $185 \mathrm{~cm}$, chose the Nana positioning protocol for 318 comfort, and did so, based on not having to actively maintain their foot in plantargrade during 319 the scan. Additionally, the Nana positioning protocols' use of pads to maintain the hands in a 
320 midprone position, allows for larger individuals (width wise) to be scanned more easily in

321 comparison to the NHANES, where the individual's hands are pronated flat on the table.

322

323 Future research needs to investigate if certain positioning protocols are more applicable for

324 different participants dependent upon their size. Furthermore, more research is required to

325 ascertain the difference between the positioning protocols when using regional analysis.

326

327 The implications for clinical practice are that the decision of which positioning protocol to

328 employ should be based on comfort, ie. the size of the participant's and not purely on the level of

329 evidence for the protocols as both protocol produce very good results. As such, the NHANES

330 protocol should be the first choice when scanning based on the comfort findings, however the

331 Nana protocol provides a fantastic alternative for larger individuals.

332

333

\section{CONCLUSION}

334 When all sources of biological and technical errors have been accounted for, the Nana and 335 NHANES positioning protocols both produce a very high level of agreement as demonstrated by 336 very high results. However, the systematic bias revealed in the limit of agreement plot and the 337 large 95\% CL indicated that the two protocols should not be used interchangeably. Anecdotally, 338 the NHANES positioning protocol was more comfortable.

\section{ADDITIONAL INFORMATION AND DECLARATIONS}

\section{Acknowledgements}


343 The authors would like to thank the 2017 graduating Physiotherapy cohort for donating their

344 time for this study.

345

346 REFERENCES

347 Australian and New Zealand Bone Mineral Society (ANZBMS). 2014. Clinical Bone Densitometry Training Course. ANZBMS; Sydney.

Australian Radiation Protection and Nuclear Safety Agency (ARPANSA). 2005. Exposure of humans to ionizing radiation for research purposes. ARPANSA, Yallambie.

351

352

353

354

355

356

357

358

359

360

361

362

363

364

365
Bilsborough JC, Greenway K, Opar D, Livingstone S, Cordy J, and Coutts AJ. 2014. The accuracy and precision of DXA for assessing body composition in team sport athletes. Journal of sports sciences 32:1821-1828. 10.1080/02640414.2014.926380

Climstein M, Pollard Z, Furness J, Walsh J, McClellan C and Meerkin J. 2015. Effects of longterm surfing on bone health in mature-aged males. International Journal of Aquatic Research and Education 9: 24-37.

Colyer SL, Roberts SP, Robinson JB, Thompson D, Stokes KA, Bilzon JL, and Salo AI. 2016. Detecting meaningful body composition changes in athletes using dual-energy x-ray absorptiometry. Physiological Measurement 37:596-609. 10.1088/0967$3334 / 37 / 4 / 596$

Covey MK, Berry JK, and Hacker ED. 2010. Regional body composition: Cross-calibration of DXA scanners QDR4500W and Discovery Wi. Obesity 18:632-637.

Covey MK, Smith DL, Berry JK, and Hacker ED. 2008. Importance of cross-calibration when replacing DXA scanners: QDR4500W and Discovery Wi. Journal of Nursing Measurement 16:155-170. 10.1891/1061-3749.16.3.155 
366 Georgeson EC, Weeks BK, McLellan CP, and Beck BR. 2011. Body composition change over a professional rugby league season and relationship to rates and types of injury. Medicine \& Science in Sports \& Exercise 43:108.

369 Kerr A, Slater GJ, Byrne N, and Nana A. 2016. Reliability of 2 different positioning protocols for dual-energy x-ray absorptiometry measurement of body composition in healthy adults. Journal of Clinical Densitometry 19:282-289. 10.1016/j.jocd.2015.08.002

372 Lee SY, and Gallagher D. 2008. Assessment methods in human body composition. Current 373 Opinion in Clinical Nutrition and Metabolic Care 11:566-572. 10.1097/MC0.0b013e32830b5f23

Lewiecki EM. 2005. Clinical applications of bone density testing for osteoporosis. Minerva medica 96:317-330.

Lexell JE, and Downham DY. 2005. How to assess the reliability of measurements in

Lohman M, Tallroth K, Kettunen JA, and Marttinen MT. 2009. Reproducibility of dualenergy x-ray absorptiometry total and regional body composition measurements Tracking fat-free mass changes in elderly men and women using single-frequency using different scanning positions and definitions of regions. Metabolism: clinical and experimental 58:1663-1668. 10.1016/j.metabol.2009.05.023 rehabilitation. Am J Phys Med Rehabil 84:719-723. 
388 Munro B, and Visintainer M. 2005. Statistical methods for health care research: Lippincott, 389 Philadelphia.

390 Nana A, Slater GJ, Hopkins WG, and Burke LM. 2012. Effects of daily activities on dual391 energy x-ray absorptiometry measurements of body composition in active people. Medicine \& Science in Sports \& Exercise 44:180-189. 10.1249/MSS.0b013e318228b60e

Nana A, Slater GJ, Hopkins WG, and Burke LM. 2013. Effects of exercise sessions on DXA 395 measurements of body composition in active people. Medicine \& Science in Sports \& Exercise 45:178-185. 10.1249/MSS.0b013e31826c9cfd

397 National Health and Nutrition Examination Survey (NHANES). 2013. Body composition procedures manual. https://www.cdc.gov/nchs/data/nhanes/nhanes 13 14/2013 Body Composition DXA.

400 pdf

401

402

403

404

405

406

407

408

409

410

Rothney MP, Brychta RJ, Schaefer EV, Chen KY, and Skarulis MC. 2009. Body composition measured by dual-energy x-ray absorptiometry half-body scans in obese adults. Obesity 17:1281-1286. 10.1038/oby.2009.14

Smith-Ryan AE, Mock MG, Ryan ED, Gerstner GR, Trexler ET, and Hirsch KR. 2016. Validity and reliability of a 4-compartment body composition model using dual energy x-ray absorptiometry-derived body volume. Clinical Nutrition. http://dx.doi.org/10.1016/j.clnu.2016.05.006

Trevethan R. 2016. Intraclass correlation coefficients: clearing the air, extending some cautions, and making some requests. Health Services and Outcomes Research Methodology:1-17. 10.1007/s10742-016-0156-6 